\section{Disclosures}

$L M K$ has received research funding and consultancy payments from Agios Pharmaceuticals and Celgene Corporation.

\section{References}

1. Hleihel R, El Hajj H, Wu H-C, et al. A Pin1/PML/P53 axis activated by retinoic acid in NPM-1c acute myeloid leukemia. Haematologica. 2021;106(12):3090-3099

2. Patel SS, Kluk MJ, Weinberg OK. NPM1 biology in myeloid neoplasia. Curr Hematol Malig Rep. 2020;15(4):350-359.

3. Schlenk RF, Döhner K, Kneba M, et al. Gene mutations and response to treatment with all-trans retinoic acid in elderly patients with acute myeloid leukemia. Results from the AMLSG Trial AML HD98B.
Haematologica. 2009:94(1):54-60.

4. Martelli MP, Gionfriddo I, Mezzasoma F, et al. Arsenic trioxide and all-trans retinoic acid target NPM1 mutant oncoprotein levels and induce apoptosis in NPM1-mutated AML cells. Blood. 2015;125(22):3455-3465.

5. El Hajj H, Dassouki Z, Berthier C, et al. Retinoic acid and arsenic trioxide trigger degradation of mutated NPM1, resulting in apoptosis of AML cells. Blood. 2015;125(22):3447-3454

6. de Thé H. Differentiation therapy revisited. Nat Rev Cancer. 2018;18(2):117-127.

7. Wei S, Kozono S, Kats L, et al. Active Pin1 is a key target of all-trans retinoic acid in acute promyelocytic leukemia and breast cancer. Nat Med. 2015;21(5):457-466.

8. Zhou XZ, Lu KP. The isomerase PIN1 controls numerous cancer-driving pathways and is a unique drug target. Nat Rev Cancer 2016;16(7):463-478.

9. Lallemand-Breitenbach V, de Thé H. PML nuclear bodies: from architecture to function. Curr Opin Cell Biol. 2018;52:154-161.

\title{
Arsenic and all-trans retinoic acid for acute promyelocytic leukemia: yes, it really is as good as it seems
}

\author{
Mark Levis \\ Department of Oncology, Johns Hopkins University, Baltimore, MD, USA \\ E-mail: MARK LEVIS - levisma@jhmi.edu
}

doi:10.3324/haematol.2021.278984

I $\mathrm{n}$ this issue of Haematologica, Kayser and colleagues report the results of an analysis of outcomes from the National Acute Promyelocytic Leukemia (APL) Observational study (NAPOLEON-Registry; NCT02192619), including 152 non-high-risk APL patients in Germany and France. ${ }^{1}$ In their study, which they claim represents a reflection of "real-life" outcomes, these authors specifically focused on APL patients treated upfront with all-trans retinoic acid (ATRA) and arsenic, according to the study led by the late Francesco Lo Coco. ${ }^{2}$ As with that original protocol, this present study excluded high-risk APL patients.

When Lo-Coco's study was published in 2013, the results seemed almost too good to be true. ${ }^{2}$ The eventfree survival rate of patients treated with ATRA and arsenic was $97 \%$. In their study of the registry patients, Kayser and colleagues found an almost identical result (event-free survival of $95 \%$, with no patient relapsing after achieving remission. The remarkable efficacy of this regimen seems to be every bit as high even outside of the context of a clinical trial. Two out of 152 patients died during induction, and both were older (64 and 70 years) than typical APL patients. Interestingly, differentiation syndrome was only reported in seven patients $(6 \%)$, in contrast to the $19 \%$ reported in Lo-Coco's study. One wonders whether this is more a reflection of clinicians' comfort in managing and even preventing this condition as they grow more familiar with this regimen over time.

Where to next with APL? Certainly, an oral version of arsenic would expand the use of this combination to many parts of the world lacking access to intravenous medication. It would also represent a major improvement in the quality of life of APL patients, who must trudge through months of daily arsenic infusions. Oral preparations are under investigation, ${ }^{3}$ but formulation challenges have thus far been an obstacle to their widespread use.
High-risk APL patients were excluded from these studies, and of course they represent a significant challenge for physicians treating them. In one of the original pilot studies exploring the combination of ATRA and arsenic, gemtuzumab ozogamycin (GO) was used as a cytoreductive agent in the high-risk patients. ${ }^{4}$ This highly effective agent is not approved for such use worldwide, but studies to compare its efficacy against anthracyclines are warranted.

Another way to potentially optimize this therapy is to determine how much arsenic is really needed to achieve these high-quality outcomes. The selection of four cycles of consolidation with arsenic was somewhat arbitrary, and no one should lose sight of the fact that arsenic is a group 1 human carcinogen with neurotoxic and hepatotoxic effects. ${ }^{5}$ Identifying the minimum necessary number of cycles would be a worthwhile endeavor for the field.

Finally, lest we be too self-congratulatory about how well we are doing with this dreadful malignancy, let us not forget how many patients die of APL before their disease is recognized and treated. ${ }^{6}$ At present, in areas of the world that have complete access to standard-of-care leukemia treatment, most APL patients die because their care providers are unknowingly observing the natural history of untreated APL. The failure to recognize APL rapidly is a problem without an immediate solution. However, perhaps in this digital age, there is a ray of hope for this problem. The use of artificial intelligence algorithms combined with digital scanning technology may offer an automated way of identifying an APL patient, ${ }^{7}$ leading to the same sort of electronic red flag that occurs when a patient with a low electrolyte or platelet count is evaluated by an emergency room physician. We are probably not far off from that future.

\section{Disclosures}

No conflicts of interest to disclose. 


\section{References}

1. Kayser S, Schlenk R, Lebon D, et al. Characteristics and outcome of patients with low-/intermediate-risk acute promyelocytic leukemia treated with arsenic trioxide - an international collaborative study. Haematologica. 2021;106(12):3100-3106.

2. Lo-Coco F, Avvisati G, Vignetti M, et al. Retinoic acid and arsenic trioxide for acute promyelocytic leukemia. $N$ Engl J Med. 2013;369(2):111-121.

3. Zhu HH, Wu DP, Du X, et al. Oral arsenic plus retinoic acid versus intravenous arsenic plus retinoic acid for non-high-risk acute promyelocytic leukaemia: a non-inferiority, randomised phase 3 trial. Lancet Oncol. 2018;19(7):871-879.
4. Estey E, Garcia-Manero G, Ferrajoli A, et al. Use of all-trans retinoic acid plus arsenic trioxide as an alternative to chemotherapy in untreated acute promyelocytic leukemia. Blood. 2006;107(9):3469-3473.

5.Zhou Q, Xi S. A review on arsenic carcinogenesis: epidemiology, metabolism, genotoxicity and epigenetic changes. Regul Toxicol Pharmacol. 2018;99:78-88.

6. Lehmann S, Ravn A, Carlsson L, et al. Continuing high early death rate in acute promyelocytic leukemia: a population-based report from the Swedish Adult Acute Leukemia Registry. Leukemia. 2011;25(7):1128-1134.

7. Sidhom JW, Siddarthan IJ, Lai BS, et al. Deep learning for diagnosis of acute promyelocytic leukemia via recognition of genomically imprinted morphologic features. NPJ Precis Oncol. 2021;5(1):38.

\section{Daratumumab: new indications revolving around "off-targets"}

\section{Yishai Ofran}

Department of Hematology, Shaare Zedek Medical Center, Faculty of Medicine, Hebrew University of Jerusalem, Israel E-mail: YISHAI OFRAN - yofran@szmc.org.il

doi:10.3324/haematol.2021.279487

T he IgG1א monoclonal anti-CD38 antibody daratumumab, approved only 5 years ago, has become a principal agent in the treatment of patients with multiple myeloma. Evidence from multiple trials and realworld experience proved its safety and effectiveness. ${ }^{1}$ Daratumumab targets CD38, a membrane glycoprotein with various functions. Binding of CD38 to its ligand (CD31) enables plasma cells to interact with surrounding immune and endothelial cells. Additionally, CD38 serves as a dual active enzyme involved in generating and hydrolyzing ADP-ribosyl cyclase and, therefore, affects intracellular calcium signaling and energy metabolism. ${ }^{2}$ Normal human plasma cells, as well as myeloma cells, express CD38 highly. The anti-myeloma effect of daratumumab is mediated through the elimination of CD38expressing plasma cells. During anti-myeloma therapy, binding of daratumumab to CD38 $8^{+}$natural killer (NK), T and B cells, and erythrocytes leads to "off-target" effects as well as to some common side effects, such as interference with blood product cross-matching or potential immunemodulation through regulatory $\mathrm{T}$-cell elimination. However, the clinical significance of "off-target" effects of daratumumab on $\mathrm{CD} 38^{+}$non-plasma cells is not yet fully characterized.

In this issue of Haematologica, Crickx and colleagues ${ }^{3}$ reported the outcome of eight patients treated with daratumumab for refractory immune thrombocytopenia (ITP) or warm autoimmune hemolytic anemia (AIHA). Patients were struggling with long-lasting diseases, with a median duration of 84.5 months (range, 18-174), refractory to multiple lines of standard therapies. The protocol of daratumumab administration was weekly infusions of $16 \mathrm{mg} / \mathrm{kg}$ combined with oral dexamethasone for at least four doses. Three out of five ITP patients and one of two patients with warm AIHA responded. A decrease in gammaglobulin levels was reported, but the autoimmune suppressive effect of daratumumab in these patients most probably went beyond its effect on patients' normal plasma cells. Notably, in addition to plasma and mature B cells, CD38 is also expressed by $\mathrm{T}$ and NK cells, and can also be induced by interferon and other cytokines. These cells are considered "off-targets" and in multiple myeloma patients treated with daratumumab, a reduction in regulatory T-cell count and expansion of $\mathrm{CD}^{+}$and $\mathrm{CD} 8^{+} \mathrm{T}$ cells were reported. ${ }^{4}$ Therefore, caution is required with co-administration of daratumumab and checkpoint inhibitors or other immune therapies. Studies are ongoing to confirm the safety of such combinations. Given its multiple targets, predicting which patients with autoimmune diseases will benefit from daratumumab is a challenge.

The patients treated by Crickx and colleagues had longlasting ITP or warm AIHA resistant to various lines of therapy. ITP and warm AIHA are antibody-mediated diseases and therefore one can speculate that daratumumab targeted mature B or plasma cells which survived previous lines of therapy. Such a mechanism may apply in other antibody-mediated refractory diseases. Indeed, reports of successful treatments in similar situations are accumulating (Table 1), including daratumumab as a therapeutic option in $\mathrm{ABO}$ mismatch-derived post-allogeneic stem cell transplantation hemolysis/cytopenia or pure red cell aplasia, antibody-mediated rejection of transplanted kidney, and even in a refractory case of antiphospholipid syndrome. An attempt to investigate the immune pathophysiology of ITP was made through a comprehensive pathology evaluation of patients' spleens. CD38 was identified as a prominent marker specifically present in clinically severe cases. ${ }^{5}$ However, despite broad-range staining for multiple markers, the authors could not definitely confirm that the $\mathrm{CD}^{3} 8^{+}$cells were of B-cell or plasma-cell phenotype.

The potential activity of daratumumab in targeting $T$ cells or early lymphoid precursors was demonstrated in a preclinical study in mice injected with T-cell acute lymphoblastic leukemia. ${ }^{6}$ Next came reports of successful treatment of patients with resistant cases of acute lymphoblastic leukemia with daratumumab, ${ }^{7.9}$ with best and lasting responses achieved in patients treated for minimal residual disease eradication. Interestingly, daratumumab was recently reported to be active in diseases in which the pathological immune response was complicated and 